\title{
Supporting information: Plasmonic trace sensing below the photon shot noise limit
}

\author{
R. C. Pooser ${ }^{1}$ \& B. J. Lawrie ${ }^{1 *}$ \\ Quantum Information Science Group, Computational Science and Engineering Division, \\ Oak Ridge National Laboratory, Oak Ridge, TN \\ E-mail: pooserrc@ornl.gov
}

Plasmonic sensors are important detectors of biochemical trace compounds, but those that utilize optical readout are approaching their absolute limits of detection as defined by the Heisenberg uncertainty principle in both differential intensity and phase readout. However, the use of more general minimum uncertainty states in the form of squeezed light can push the noise floor in these sensors below the shot noise limit (SNL) in one analysis variable at the expense of another. Here, we demonstrate a quantum plasmonic sensor whose noise floor is reduced below the SNL in order to perform index of refraction measurements with sensitivities unobtainable with classical plasmonic sensors. The increased signal to noise ratio (SNR) can result in faster detection of analyte concentrations that were previously lost in the noise. These benefits are the hallmarks of a sensor exploiting quantum readout fields in order to manipulate the limits of the Heisenberg uncertainty principle.

\section{Nonlinear amplifier field equations and squeezed states}

Quantum noise reduction, or "squeezing" is defined as the ratio of the noise in a quantum state to the shot noise, or the noise of a coherent state, which is a minimum uncertainty 
state with noise dictated by the Heisenberg uncertainty principle. A coherent state saturates the Heisenberg inequality, $\Delta x \Delta p=\hbar / 2$, with $\Delta x=\Delta p$. It is common to set $\hbar / 2=1$ when writing the noise in units of shot noise. A squeezed state is also a minimum uncertainty state, but allows for a more general form with $\Delta x \Delta p=1$ and $\Delta x=\frac{1}{\Delta p}$. Squeezed states can be generated using quantum optics techniques, such as pumping a nonlinear medium to act as a nonlinear amplifier or oscillator. Squeezing can be generalized to more than one optical mode if the output of the nonlinear medium is nondegenerate. In this case, the difference between the two fields' intensities can be squeezed, for instance.

The quantum noise of a nonlinear amplifier is well-illustrated in the interaction frame of the Heisenberg picture. In this frame, the interaction Hamiltonian for a third order nonlinear amplifier (which best describes the quantum light source used in our experiment) is

$$
H=i \hbar \chi^{(3)} a_{p r} a_{c} a_{p}^{\dagger} a_{p}^{\dagger}+H . C .
$$

where $\chi^{(3)}$ is the nonlinear coefficient and $a_{p}$ is the pump field amplitude, which is assumed to be undepleted. The Heisenberg equations of motion are found by taking the commutator of the field operators with the Hamiltonian:

$$
\begin{aligned}
& \dot{a}_{p r}=\kappa a_{c}^{\dagger} ; \\
& \dot{a}_{c}=\kappa a_{p r}^{\dagger} .
\end{aligned}
$$

The equations can be decoupled by examining the second time derivative, which yields exponential solutions :

$$
\begin{aligned}
& a_{p r}(t)=a_{p r}(0) \sqrt{G}-a_{c}(0)^{\dagger} \sqrt{G-1} \\
& a_{c}(t)^{\dagger}=a_{c}(0)^{\dagger} \sqrt{G}-a_{p r}(0) \sqrt{G-1}
\end{aligned}
$$

where $\kappa$ is the combined nonlinearity multiplied by the pump amplitude, which has been 
taken to be a classical number, and $\sqrt{G}=\cosh \kappa t$, and $t$ represents the interaction time in which the fields traverse the nonlinear medium. Given these operators, one can predict the expectation value for the photon number in each field after the nonlinear process, along with the corresponding noise:

$$
\begin{array}{r}
N_{p r}=G \alpha^{*} \alpha \\
\Delta N_{p r}^{2}=G \alpha^{*} \alpha \\
N_{c}=(G-1) \alpha^{*} \alpha \\
\Delta N_{c}^{2}=(G-1) \alpha^{*} \alpha,
\end{array}
$$

where $\alpha$ is the input field to the amplifier $p r$ port. While each field has excess noise added that scales as $G$, the noise on the photon number difference is

$$
\Delta N_{-}^{2}=\alpha^{*} \alpha
$$

if a perfect detector is used to detect the two fields. After normalization to the total output noise, the difference noise becomes

$$
\Delta N_{-, n}^{2}=\frac{1}{(2 G-1)}
$$

The noise in Eq. 11 is normalized to the SNL (where $n$ denotes normalization). In the absence of a nonlinear amplifier $(G=1)$, the total output noise is a single shot noise unit. The total noise is inversely proportional to $G$ if both fields are detected with perfect detectors, as illustrated in Fig. 1. However, if the fields are attenuated, the field operators used in the difference operator change according to an effective beam splitter model with total transmission $\eta$ on the probe and conjugate beams.

$$
a_{p r_{\text {out }}}=\sqrt{G \eta} a_{p r_{\text {in }}}+\sqrt{(G-1) \eta} a_{c_{\text {in }}}^{\dagger}+\sqrt{(1-\eta)} a_{v d 1}
$$




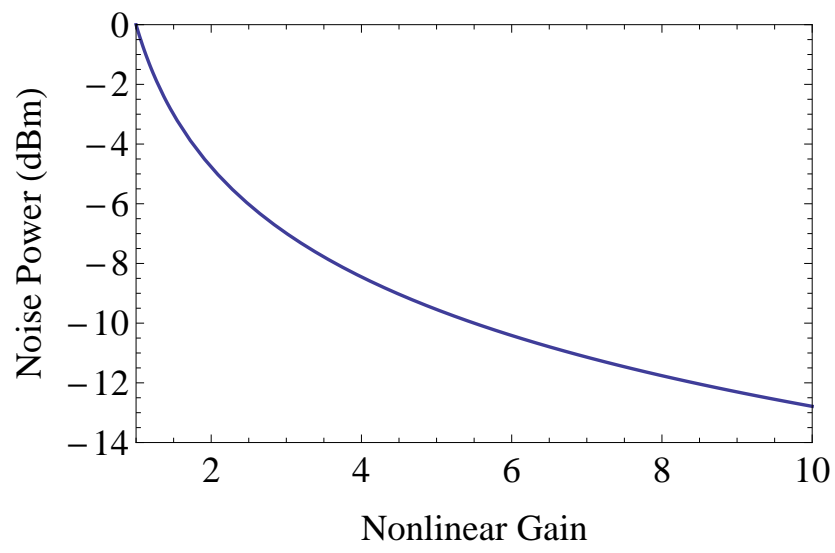

Figure 1: Quantum noise reduction as a function of nonlinear gain without losses plotted on a logarithmic scale.

$$
a_{c_{\text {out }}}=\sqrt{G \eta} a_{c_{\text {in }}}+\sqrt{(G-1) \eta} a_{p r_{\text {in }}}^{\dagger}+\sqrt{1-\eta} a_{v d 2}
$$

where $a_{v d i}$ are the vacuum field modes that interfere with each field at its respective beam splitter and $\eta$ is the effective beam splitter transmission. In this case the noise becomes

$$
\Delta N_{-, n}^{2}=1+\frac{2 \eta(1-G)}{2 G-1} .
$$

The noise as a function of loss for $G=4$ is shown in Fig. 2 .

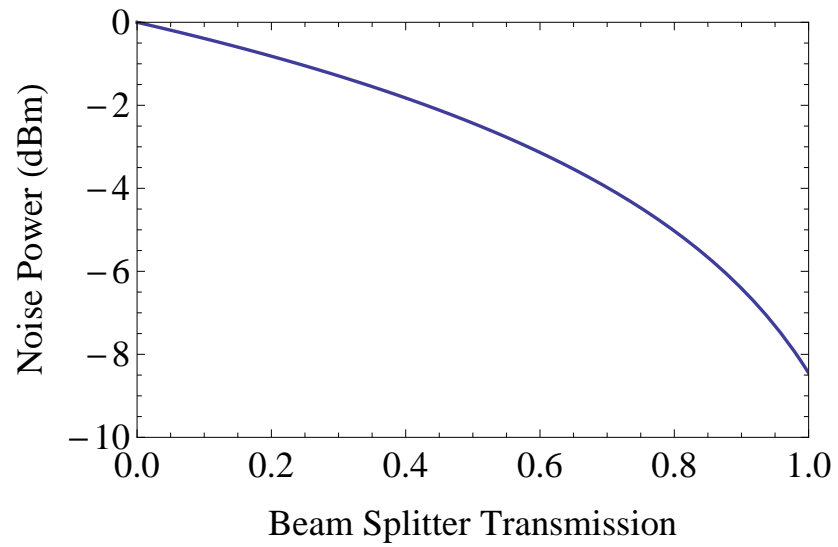

Figure 2: Quantum noise reduction as a function of equal losses on both the probe and conjugate fields for nonlinear gain $G=4$. This accounts for imperfect detection efficiency of the two fields on identical detectors. 